\title{
Promoting analytical quality control of trace-element data to be presented in international journals and reports
}

\author{
Lars Jorhem
}

Received: 21 February 2008/ Accepted: 30 April 2008/Published online: 27 May 2008

(C) Springer-Verlag 2008

\begin{abstract}
The analytical quality of published data on trace elements in food has previously been shown to be of a generally poor quality and, therefore, not always reliable. The responsibility for this problem is shared among authors, editors, reviewers, and publishers, and it is based on the lack of clear instructions, to all parties, on how quality should be safeguarded. This has been noted by CEN/TC 275/WG 10, which has started preliminary work on such a guideline. This paper describes the background and what such a guideline could be based on.
\end{abstract}

Keywords Analytical quality - Quality control ·

Guideline · CEN/TC 275/WG 10

\section{Introduction}

This paper refers to trace elements and heavy metals. Although neither term is officially defined they are rooted in the general consciousness and in the scientific community. Whereas trace elements usually indicate something positive, heavy metals are mostly associated with toxic elements, irrespective of mass, but there is no sharp boundary between the terms. Their use in this paper is not an attempt to endorse the terms. It is only a matter of simplicity.

In ISO 17025 it is stated that "The laboratory shall have quality control procedures for monitoring the validity of tests and calibrations undertaken" [1]. In this paper quality control is given a somewhat wider definition: If the

\section{Jorhem ( $\square)$}

Convenor of CEN/TC 275/WG10,

National Food Administration, R\&D/K2,

Box 622, 75126 Uppsala, Sweden

e-mail: lajo@slv.se analytical work and the results obtained are described in a journal in such a way that the reader can conclude that the analyst/author is competent within the analytical area and realises the importance of describing the use of relevant quality-control tools, then the paper is of good quality (that helps the reader to validate the presented data).

Consequently, if a paper lacks all, or most, of the attributes that help the reader to validate the content of the paper it is of poor quality, in which case the analytical data cannot be relied upon.

The state of the art of analytical quality

In a paper published 2006 [2] it was concluded that analytical quality control (AQC) of data published in papers on trace element analysis in food is rather poor. This conclusion was based on the finding that many papers showed vast ranges and high-individual results of, e.g., lead or cadmium in samples that were not suspected of being contaminated prior to sampling or during sample handling. After having scrutinised 105 papers it became rather clear that the AQC in the reports is often inconsistent, difficult to find and understand, and sometimes completely missing. Papers that had no, or limited, description of the analytical quality-control procedures often covered wide ranges and/or contained inexplicably high (individual) results. Papers with a thorough description of the analytical quality never had this type of distribution of results. Statistical evaluation showed there is a relationship between the quantity of AQC described and the span of results, that is as AQC descriptions grow, the range of results in individual surveys tends to decrease.

One conclusion from this was that authors who do write good AQC descriptions are also competent analysts, whereas those who do not are, occasionally, probably less competent. 
It was also concluded that description of the analytical quality had not generally improved over the last decade. This is surprising, considering the increased importance given to analytical quality assurance (AQA) and accreditation.

It is generally assumed that the quality of analytical data presented in national or international journals is reliable. This is probably especially true for peer-reviewed journals. There is, however, very little reason for such confidence. There are several pitfalls on the way:

- Potential authors are not aware of the need for stringent description of analytical quality control and find little or no guidance in the "instructions for authors" found in most journals (there are exceptions), because these focus on the style and the content of submitted papers and do not give much information to the authors about how analytical quality should be presented and safeguarded. This probably stems from the wrong assumption that all authors are highly competent analysts.

- When a manuscript is submitted to a journal it is either dealt with at the editorial office, or sent for peer reviewing. The instructions to the reviewer focus on checking the manuscript's relevance for the journal and that the manuscript complies with editorial and statistical requirements and guidelines. Very little focus, if any, is put on the analytical quality of the data. The reviewer is usually selected according to competence and can from personal experience judge whether or not the results seem reasonable, and advise the author accordingly. But, because it can be difficult to find reviewers with specialist competence, the request may go to someone in a more or less distant area, in which case the quality judgement will be more difficult. It is probably a fair guess that many reviewers do not want, or do not have the competence, to be too critical of the analytical quality in a paper.

- The editor/editorial office faces similar problems; those with specialist competence may be able to spot extreme or improbable results, others will not. Another troubling factor is that some journals actively discourage authors from including analytical quality information in their papers. Editors may also be under pressure to find enough papers to publish and may not always be too critical about the quality control.

Based on the findings described above, it is probably safe to say that the state of the art of published analytical data is less than satisfactory. As a result the reliability and trueness of the results are not always safeguarded. Analytical data with a questionable quality appear alongside data of satisfactory quality. A problem is that the reader may not be able to distinguish between the two. Therefore results from unreliable publications may find their way into legislation and intake recommendations for food.
Jenks and Stoeppler [3] concluded in 2001 that description of the use of certified reference materials (CRMs) for AQC is far from satisfactory. In addition they found that analytical quality control procedures, described in publications, do not follow any template. Fragments of the quality description can in principle be found anywhere in the papers, which obviously makes it more difficult to acquire a comprehensive idea of the analytical quality. One of their conclusions was that "publishers should set distinct and agreed recommendations that define where and how the description of CRMs used should be mentioned".

These problems have been discussed within working group (WG) 10 (trace elements and heavy metals) of CEN/ TC 275. A conclusion from these discussions was that there is no systematic approach to the publication of analytical data: There are no general guidelines to authors, reviewers, or editors on how to judge analytical quality. It was therefore suggested that WG 10 bring this up as a new work item, with the objective of developing a guideline/standard that would promote the use of relevant analytical-quality control data that could be useful for all parties involved in the publication of analytical data. This suggestion was accepted by TC 275 .

\section{The description of analytical quality}

Analytical quality control is, or should be, an integral part of all analytical processes. It is an integral part of the AQA programme that may form the basis for method accreditation. Without AQC it cannot be known what the results actually represent. What analysts perceive individually as analytical quality may vary substantially. A traditional approach is to analyse a spiked sample and check how much remains, the recovery. In some areas of chemistry this is an important step in terms of analytical quality, for example for methods which contain an extraction step, whereas in other areas, such as the total quantification of metals in food, it is not.

Is accreditation the ultimate proof of analytical proficiency? There are indications to the contrary. In the field of trace element analysis, the IMEP (International Measurement Evaluation Programme) for proficiency testing [4, 5] has indicated that the distribution of results, for example for $\mathrm{Pb}$, from laboratories with and without accreditation follow the same pattern. It can thus be concluded that a claim by an author that the method used is accredited does not by itself give any guarantee of the quality/reliability of the presented results.

ISO 17025 [1] indicates that you should use certified reference materials, when available, and/or take part in proficiency testing (PT), when suitable programmes are available. It has been shown that CRMs are not always used, and that when they are used they are not always used 
correctly, evaluation of the results often is performed haphazardly, and there is frequently poor matching of CRM and sample with regard to matrix and analyte concentration [6-8]. Furthermore, when looking through papers containing data on CRMs it is striking how unusual it is to find a "non-satisfactory" result. This could indicate that international analytical competence is generally very good. The results from PT programmes, however, give a completely different picture. In trace element programmes, e.g. those of FAPAS [9] or the NFA [10], it is not unusual for up to $50 \%$ of the participants to have $z$-scores outside the range \pm 2 ; which is not satisfactory. It should be noted that the results from PT programmes are almost never published, either individually or alongside CRM results.

There are many important factors in analytical quality control that affect the credibility of published results, for example:

- do the authors show awareness of analytical problems in the described procedure,

- how do they handle blanks and the limit of detection,

- have they described comprehensively how sampling was done, how samples were stored, and how samples were pre-treated,

- are unusual/unexpected results followed by, for example, repeated analysis, or commented on convincingly?

\section{The content of a possible guideline/standard}

A non-mandatory guideline/standard will probably remain unused if its usefulness is not obvious to the target groups, in these case authors of reports and journals, journal editors, and reviewers assigned by editors. To meet such demands a guideline must be simple, comprehensive, and self explanatory. A common checklist/reporting form for writers, editors, and reviewers may be a starting point.

The analytical quality criteria listed below are taken from Ref. [1], where they were used for the evaluation of the content of a large number of published papers. These criteria could be a starting point for further elaboration and discussion on what should be the framework of a guideline/ standard. It is not likely that an author would be able to comply with every criterion, but the more the better, and some criteria are probably more important than others.

Please note that these criteria were developed for trace element analysis and may need some modification in order to be generally applicable.

\section{Choice of method and method description}

This is a fundamental aspect of quality for both an author and a reviewer and rather simple to evaluate. If an author has used an apparently unsuitable method, but explains why it was chosen, a reviewer may judge whether it is, nevertheless, acceptable for the analytical purpose. If it is not explained the reviewer may need to reject the paper.

Awareness of interferences

If an author has not shown that he is aware of the pitfalls present in the method/technique used it is very possible he is not aware of them, which could be very serious in, for example, trace element analysis. This is, therefore, a fundamental aspect for a reviewer/editor to judge.

Determination of sample blanks

This may be highly important. The reviewer must judge if they are significant and how they should be handled. The blank may also provide the basis for the limit of detection, if relevant to the concentration. This may also require a definition of the limit of detection.

Certified reference materials

Are CRMs used? If so, are they relevant and in respect of the matrix and concentration concerned?

Proficiency testing

This is the only independent way of judging someone's analytical competence. The presentation of PT results should therefore be mandatory in papers presenting analytical results, if a programme is available. If no programme is available, this should be stated clearly. Again, relevant matrix and concentration is important.

\section{Measurement uncertainty}

This is still a hot topic (or at least lukewarm) and it is probably just a matter of time before its reporting becomes mandatory.

\section{Recovery}

Its importance is minor and mostly of no importance in methods for the determination of trace elements using standard methods. It may, however, be used for additional information.

Probability of contamination of the samples before or after arriving in the laboratory

This is very important, but how can it be described more generally? In addition, sampling is mostly outside the 
control of the laboratory staff. It is mainly a problem in trace element analysis. Perhaps there should be a general statement that "the reviewer should consider if unusually high results could be due to inadvertent contamination during the sampling process or the sample handling" or something to that effect.

\section{Realisation of the project}

As already mentioned this is a project initiated and run by CEN/TC 275/WG 10, whose members have wide experience, especially in trace element analysis but also in other areas, which will be very important if/when this proposal for a guideline is widened to cover most parts of analytical chemistry. A first draft for a guideline is currently being written and is expected to be available at the next meeting of the WG 10 in November 2008. At a later stage it will probably involve other working groups within TC 275 . If it is then concluded that a guideline is feasible for food chemistry in general, it is possible the project will be completed around 2010-2011.

Many of the members of the WG also act as reviewers for various journals, which provides further important competence for this project.

Furthermore, in order for competence to be as broad as possible in this project, several editors of well known journals with high quality standards are connected to the project. They serve as a sounding board and to ensure that the final product is relevant for the journal also.
Acknowledgments The ad hoc group from CEN/TC 275/WG 10 primarily involved in the development of a first draft consists of: Kåre Julshamn, National Institute of Nutrition and Seafood Research, PO Box 2029 Nordnes, N-5817 Bergen, Norway; Jörg Oehlenshläger, Federal Research Centre for Nutrition and Food, Department for Seafood Research, Palmaille 9, 22767 Hamburg, Germany; Henk van der Schee, Food and Consumer Product Safety Authority (VWA), Hoogte Kadijk 401, NL-1018 BK, Amsterdam, The Netherlands; and Joakim Engman, National Food Administration, Box 622, SE-751 26 Uppsala, Sweden

\section{References}

1. ISO/IEC 17025 (2005) General Requirements for the Competence of Calibration and Testing Laboratories. International Organisation for Standardisation. Geneva, Switzerland

2. Jorhem L, Engman J, Sundström B, Nilsson A (2006) Accred Qual Assur 10:647-658

3. Jenks P, Stoeppler M (2001) Fresenius J Anal Chem 370:164169

4. European Commission, Joint Research Centre, Institute for Reference Materials and Measurements (2003) IMEP-19 Trace Elements in Rice EUR 20551 EN Report to Participants

5. European Commission, Joint Research Centre, Institute for Reference Materials and Measurements (2004) IMEP-20 Trace Elements in Tuna fish EUR 21018 EN Report to Participants

6. Jorhem L (1998) Fresenius J Anal Chem 360:370-373

7. Jorhem L (2004) Accred Qual Assur 9:305-310

8. Jorhem L (2004) Accred Qual Assur 9:507-508

9. FAPAS (Food Analysis Performance Assessment Scheme) Central Science Laboratory Sand Hutton, York, UK, YO41 1LZ

10. National Food Administration. Proficiency testing programme for trace elements in food. Box 622, SE-751 26 Uppsala, Sweden 